\title{
Daptomycin approved in Japan for the treatment of methicillin-resistant Staphylococcus aureus
}

This article was published in the following Dove Press journal:

Therapeutics and Clinical Risk Management

16 February 2012

Number of times this article has been viewed

\author{
Mao Hagihara' \\ Takumi Umemura' \\ Takeshi Mori ${ }^{1,2}$ \\ Hiroshige Mikamo' \\ 'Department of Infection Control \\ and Prevention, Aichi Medical \\ University School of Medicine, \\ Nagakute, Aichi, Japan; ${ }^{2}$ Division \\ of Pharmaceutical Science, Faculty \\ of Pharmacy, Meijo University, \\ Nagoya, Aichi, Japan
}

Correspondence: Mao Hagihara Department of Infection Control and Prevention, Aichi Medical University School of Medicine, Nagakute, Aichi, 480-II95, Japan

Tel $+8|56| 6233 \mid I(2353)$

Fax +8I 56I 6I I842

Email hagimao@hotmail.com

\begin{abstract}
Daptomycin is a lipoglycopeptide antibacterial drug that is rapidly bactericidal for methicillin-resistant Staphylococcus aureus (MRSA) infection and has antibiotic activity against a wide range of Gram-positive organisms. It has been approved by the Ministry of Health, Labor and Welfare in Japan for the treatment for bacteremia, right-sided endocarditis, and skin and skin-structure infections, such as necrotizing fasciitis, due to MRSA on the basis of a Phase III trial conducted in Japan since July, 2011. In Japanese Phase I and III trials, daptomycin therapy given at $4 \mathrm{mg} / \mathrm{kg}$ and $6 \mathrm{mg} / \mathrm{kg}$ once per day was well tolerated and effective as standard therapy for the treatment of acute bacterial skin and skin-structure infections and bacteremia caused by MRSA, but side effects remain to be evaluated in large-scale trials.

Keywords: daptomycin, methicillin-resistant Staphylococcus aureus (MRSA), Japan
\end{abstract}

\section{The problem of MRSA in Japan}

Methicillin-resistant Staphylococcus aureus (MRSA) has spread among hospital isolates since the 1960s and these strains eventually disseminated worldwide. MRSA has long been recognized as one of the major human pathogens responsible for some afflictions such as skin and wound infections, bacteremia and endocarditis, infections of the central nervous system, respiratory and urinary tracts, and infections associated with intravascular devices and foreign bodies, and shown high mortality and morbidity. ${ }^{1}$ In Japan, MRSA primarily causes nosocomial infections. Four hundred and eighty-seven hospitals participated in the Japan Nosocomial Infection Surveillance (JANIS) system performed by the Japanese Ministry of Health, Labor and Welfare. The recorded MRSA prevalence among S. aureus isolates was 57.6\% (100845/175145 strains) in $2010 .^{2}$ This result was similar to the results from the National Nosocomial Infections Surveillance (NNIS) in the USA $(52.9 \%$ in intensive care units [ICU]; $46.0 \%$ in non-ICU wards). ${ }^{3}$

The recent emergence of decreased susceptibility to vancomycin, as the standard treatment for invasive MRSA infection due to its low cost and extensive experience with MRSA phenotypes, such as vancomycin minimum inhibitory concentrations (MICs) of $2 \mu \mathrm{g} / \mathrm{mL}$, heterogeneous vancomycin-intermediate S. aureus (hVISA), and vancomycin-intermediate $S$. aureus (VISA), presents a significant correlation with the mortality of patients infected with these isolates. ${ }^{46}$ Takesue et al compared several characteristics of 128 episodes of Japanese MRSA bacteremia between 2005 and 2008 with 631 other MRSA infections. Consequently, the clinical efficacy as first-line therapy in patients infected with $2 \mu \mathrm{g} / \mathrm{mL}$ strains was significantly lower than that for patients 
infected with $\leq 1 \mu \mathrm{g} / \mathrm{mL}$ strains ( $30.0 \%$ vs $78.8 \% ; P<0.001$ ) in bacteremia; mortality was significantly higher in patients with $2 \mu \mathrm{g} / \mathrm{mL}$ strains than in patients with $\leq 1 \mu \mathrm{g} / \mathrm{mL}$ strains (65.8\% vs $19.5 \% ; P<0.001){ }^{7}$

While some in vitro studies had already suggested that combination therapies of glycopeptides and $\beta$-lactams show synergistic effects for MRSA phenotypes and decreased vancomycin susceptibility, ${ }^{8}$ Hatano et al proposed that the existence of MRSA strains showing antagonistic effects for the combination therapy should be called $\beta$-lactam antibioticinduced vancomycin-resistant MRSA (BIVR). ${ }^{9}$ In the epidemiological investigation including two university hospitals, one hospital with 800 beds, and four hospitals with 300-500 beds in Japan, ${ }^{10}$ the BIVR detection rate was $6.7 \%$ (45 in 717 clinical MRSA isolates). Likewise, 11 linezolid (LZD)resistant clinically isolated MRSA with MICs of $>4 \mu \mathrm{g} / \mathrm{mL}$ from 11 patients at six hospitals in Japan were collected from 2006 through 2008. Alternative vancomycin-resistant strains have also been reported in Japan, while their incidences in clinical isolates are still as low as in the USA. ${ }^{11,12}$

Moreover, MRSA infections are no longer confined to health care institutions. MRSA strains isolated from community-acquired infections are becoming increasingly common. Recent research suggests that the virulence of community-acquired MRSA (CA-MRSA) infection is at least partially due to overexpression of toxins, such as Panton-Valentine leucocidin (PVL), $\alpha$-toxin, and toxic shock syndrome toxin (TSST-1) ${ }^{13,14}$ and subsequent host inflammatory response. ${ }^{15}$ In Japan, CA-MRSAs such as pulsed-field type USA300 MRSA strain infections producing PVL have also been reported since $1970-1980,{ }^{16}$ while the detection rate of CA-MRSA strains remains less than in the USA. ${ }^{17}$

\section{Overview of the current options for the treatment of MRSA}

Increasing vancomycin-resistant MRSA strains in conjunction with availability of new antibiotics, including daptomycin, have increased treatment choices but made clinical treatment decisions more challenging. Nowadays, alternative options for the treatment of MRSA infections in Japan are: the glycopeptides, teicoplanin; the oxazolidinone, LZD; the cyclic lipopeptide, daptomycin; and the aminoglycoside, arbekacin. Other agents with potential activity against MRSA are quinupristin-dalfopristin, trimethoprimsulfamethoxazole, clindamycin, erythromycin, tetracycline, rifampicin, and the fluoroquinolones. However, the use of these latter agents is generally restricted to cases of noninvasive $S$. aureus infections or is avoided because of widespread resistance, and they cannot be recommended for the treatment of invasive disease.

Although vancomycin is almost universally accepted as the drug of choice for the treatment of most MRSA infections, some shortcomings have been recognized, such as being less rapidly bactericidal compared with $\beta$-lactams against $S$. aureus, especially at higher inoculums, ${ }^{18}$ poor tissue and intracellular penetration, lack of activity against organisms growing in biofilm, and lack of interference with toxin production. The recent emergence of decreased vancomycin susceptibility in $S$. aureus, including isolates with vancomycin mininum inhibitory concentrations (MICs), presents a significant clinical problem.

Teicoplanin shows a spectrum of activity similar to that of vancomycin, to which it is closely related, and also has a mechanism of action similar to that of vancomycin. Compared with vancomycin, teicoplanin has a relatively long half-life and better tissue penetration due to its high proteinbinding potency. ${ }^{19}$ Clinical data reported that an early loading dose of teicoplanin achieves a good therapeutic effect..$^{20,21}$ Comparative meta-analysis of the clinical effects of teicoplanin and vancomycin included 24 studies (2610 patients) and suggested that, while both antibiotics achieved the equivalent clinical cure (risk ratio [RR]. 1.03; 95\% confidence interval [CI]: 0.98-1.08) and microbiological cure (RR, 0.98; 95\% CI: $0.93-1.03)$, teicoplanin has less nephrotoxic potential than vancomycin (RR, $0.51 ; 95 \%$ CI: $0.30-0.88) .^{22}$

Linezolid has good tissue migration, does not need dosage titration for kidney function and therapeutic drug monitoring, and can be switched from intravenous to oral due to having preparation and oral forms. The major adverse event associated with LZD treatment is reversible myelosuppression, mostly thrombocytopenia. The incidence of LZD-induced thrombocytopenia was higher in patients with renal failure than in patients with normal renal function, although the underlying mechanisms of this toxicity are still unknown. ${ }^{23}$ A meta-analysis including nine relevant randomized, controlled trials studied 2489 clinically assessed patients and found that LZD showed higher clinical effect for skin and soft tissue infection (odds ratio [OR], 1.40; 95\% CI: 1.01-1.95), while it showed equivalent therapeutic effect with vancomycin for bacteremia (OR, 0.88; 95\% CI: 0.49-1.58), pneumonia (OR, 1.16 ; $95 \%$ CI: $0.85-1.57$ ) patients. ${ }^{24}$ In contrast, there was no difference in total adverse effects (OR, 1.14; 95\% CI: 0.82-1.59).

Arbekacin excels in bactericidal activity against MRSA as well as daptomycin, and also has antibacterial activity for Pseudomonas aeruginosa. For more than 10 years in 
Japan, arbekacin has been successfully used to treat MRSA infections. However, due to its poor penetration and adverse effects such as nephrotoxicity and ototoxicity, arbekacin is rarely used as first-line therapy for MRSA infections. ${ }^{25}$

Daptomycin is a new lipoglycopeptide antibacterial drug that is rapidly bacterial for MRSA infection. It was approved for the treatment for bacteremia, right-sided endocarditis, and skin and skin-structure infections such as necrotizing fasciitis due to MRSA on the basis of a Phase III trial conducted in Japan that revealed that daptomycin therapy was noninferior to vancomycin therapy for this indication. In the early studies of daptomycin use in dogs, skeletal muscle toxicity was observed frequently, as measured by elevations of the creatinine phosphokinase (CPK) muscle isoenzyme, and the experiments revealed that once-daily dosing is much less frequently associated with this adverse effect. ${ }^{26}$

\section{Pharmacology of daptomycin Mode of action}

The antibacterial activity of daptomycin is believed to be mediated through depolarization of the bacterial cell membrane. The hydrophobic tail of the daptomycin molecule is inserted into and irreversibly binds within the bacterial cell membrane in a calcium-dependent process. ${ }^{27}$ Subsequent oligomerization of the molecules within the membrane leads to the formation of pores or ion channels, which allow potassium efflux from the bacterial cytoplasm, thus destroying the ion concentration gradient; ${ }^{28}$ this depolarizes the cell membrane and causes rapid cell death through the inhibition of DNA, RNA, and protein synthesis. Additionally, a recent study suggests that potassium leakage is not the only mechanism: magnesium and adenosine triphosphate loss may also play a role. ${ }^{29}$

\section{Antibacterial spectrum}

Daptomycin is active against Gram-positive bacteria. Its spectrum includes staphylococci, streptococci including Streptococcus pneumoniae, and enterococci (including vancomycin-resistant strains). ${ }^{30}$ It is also active against some anaerobic organisms, such as peptostreptococci and clostridia, including Clostridium difficile. ${ }^{31}$ However, daptomycin shows no activity against Gram-negative bacteria.

\section{Resistance}

Although the mechanism of resistance is not clear, single mutations in mpr $F$ or $y y C G$, the lysylphosphatidylglycerol synthetase gene, are often presented in such strains. ${ }^{32,33}$ Julian et al revealed the change of the muropeptide profile with these strains. The daptomycin-nonsusceptible VISA's cell wall demonstrated a reduction in muramic acid $O$-acetylation, a phenotypic parameter not previously reported for VISA. ${ }^{33}$ Moreover, Muthaiyan et al suggested that the expression profile indicated that cell wall stress stimulon member genes were significantly induced by daptomycin and by the cell wall-active antibiotics vancomycin and oxacillin. They compared the daptomycin response of a two-component cell wall stress stimulon regulator VraSR mutant, S. aureus KVR, to its parent N315 and found diminished expression of the cell wall stress stimulon in the mutant. ${ }^{34}$

Prior exposures to vancomycin and elevated vancomycin MICs have been associated with increases in daptomycin MICs, suggesting possible cross-resistance. ${ }^{35-37}$ The population analysis of some MRSA phenotypes with vancomycin MIC $1-8 \mu \mathrm{g} / \mathrm{mL}$ in blood from infected patients revealed that treatment with vancomycin was associated with the development of vancomycin heterogeneous resistance and was accompanied by daptomycin heteroresistance. ${ }^{36}$ In MRSA, including VISA, physiologic changes or cell wall changes may interfere with daptomycin activity by decreasing the ability of daptomycin, a relatively large molecule, to access relevant binding regions on the bacterial cell membrane.

\section{Pharmacokinetic properties}

In Japan, the approved dose of daptomycin is $4-6 \mathrm{mg} / \mathrm{kg}$ every 24 hours. In the Japanese Phase I trial, healthy subjects $(\mathrm{n}=6)$ received single doses of 2 to $12 \mathrm{mg} / \mathrm{kg}$ of daptomycin by 30 -minute intravenous infusion. Daptomycin showed liner pharmacokinetics with a half-life of 7.4-10.2 hours and $73.4 \%$ was excreted in the urine in an unmetabolized form. One week after daptomycin dosing of 4, 6 and $10 \mathrm{mg} / \mathrm{kg}$ every 24 hours, 24-hour area under the curve $\left(\mathrm{AUC}_{0-24}\right)$ values at steady state from healthy volunteers were $424.7 \mu \mathrm{g} *$ hour $/ \mathrm{mL}$ (95\% CI: 392.5-459.5), $601.4 \mu \mathrm{g} *$ hour $/ \mathrm{mL}$ (95\% CI: 555.8 650.7), and 1094.6 $\mu \mathrm{g}^{*}$ hour/mL (95\% CI: 1011.6-1184.4), respectively. The volume of distribution at steady state in healthy subjects was low $(0.1 \mathrm{~L} / \mathrm{kg})$, indicating that it remains primarily in plasma and interstitial fluid. Although protein binding to plasma protein was high $(90 \%-93 \%)$, it decreased in patients with a creatinine clearance of $<30 \mathrm{~mL} /$ minute and was reversible, therefore protein-bound daptomycin is bioavailable and is independent of the drug concentration. However, the percentage of free drug is not a good predictor of biological effect for daptomycin. ${ }^{38}$

Furthermore, as daptomycin does not interact with the P450 cytochromes, there are no interactions with other drugs via this mechanism. ${ }^{39}$ Daptomycin is inactivated by 
surfactant in the lung and is not indicated for the treatment of pneumonia, ${ }^{40,41}$ and is not to be used to treat pneumonia and left-sided endocarditis.

\section{Pharmacodynamics of daptomycin Japanese clinical Phase III trial Study design}

The Japanese clinical Phase III trial was conducted as a randomized, open-label, active-comparator, parallel-group, multicenter study at 61 institutions from 2008 through $2010 .^{42,43}$ Eligible patients were aged over 20 years. Primary inclusion criteria were bacteremia with or without right-sided endocarditis and acute bacterial skin and skin-structure infections (ABSSSI), including wound infections (eg, surgical and traumatic wounds), major abscesses, infected diabetic ulcers of extremity, and infected ulcers due to other causes (eg, ulcers associated with vascular insufficiency or decubiti) that were due to MRSA and required hospitalization. Patients with ABSSSI were randomized 4:1 to receive intravenous daptomycin ( $4 \mathrm{mg} / \mathrm{kg}$ ) once-daily or vancomycin ( $1 \mathrm{~g})$ twice daily for 7-14 days. There was no comparative arm set for bacteremia with or without right-side endocarditis, and thus all patients received intravenous daptomycin $6 \mathrm{mg} / \mathrm{kg}$ once daily over 30 minutes for 14-42 days.

Outcomes were based primarily on clinical and microbiologic assessments, compared with baseline (within 48 hours before receipt of the first dose of study drug). Clinical assessments of signs and symptoms were recorded and assessed for potential adverse events during study therapy. Efficacy was assessed at the end of therapy and at 7-14 days (in ABSSSI patients) or 38-46 days (in bacteremia with or without right-side endocarditis) of the last dose of study medication for test-of-cure. The coprimary efficacy endpoints were the clinical and microbiologic response at test-of-cure. All assessments were reassessed by the Efficacy Adjudication Committee. Successful clinical response was defined as the clinical assessment of "cure" or "improved" at both the end of therapy and test-of-cure; not receiving a nonstudy antibacterial agent with potency against causative pathogen; receiving study drugs for at least 4 (ABSSSI group) or 7 (bacteremia with or without right-side endocarditis group) days; and a negative blood culture at test-of-cure for the causative pathogen (bacteremia with or without right-side endocarditis group). A successful microbiologic response was defined as eradication or presumed eradication of the pathogen.

For the safety analysis, the adverse events and concomitant medications were monitored daily. Vital signs and clinical laboratory parameters, including clinical chemistry, hematology, and urinalysis findings, were assessed at each scheduled evaluation.

\section{Susceptibility}

Across the Phase III trial, 121 patients were enrolled and received $\geq 1$ dose of study medication; these patients constituted 110 ABSSSI patients and 11 bacteremia with or without right-side endocarditis patients. MICs of baseline 78 isolates from patients who participated in the Phase III clinical trial against anti-MRSA agents were measured by broth microdilution method according to the Clinical and Laboratory Standards Institute testing guidelines. ${ }^{44}$ The $\mathrm{MIC}_{50}$ and $\mathrm{MIC}_{90}$ values against MRSA strains were isolated for daptomycin, vancomycin, teicoplanin, arbekacin, and LZD and were $0.5 / 0.5$ (range, $0.25-1 \mu \mathrm{g} / \mathrm{mL}$ ), $1 / 1$ (0.5-2 $\mu \mathrm{g} / \mathrm{mL}), 1 / 4(0.25-8 \mu \mathrm{g} / \mathrm{mL}), 1 / 2(0.25-4 \mu \mathrm{g} / \mathrm{mL})$, $2 / 2(1-4 \mu \mathrm{g} / \mathrm{mL}) \mu \mathrm{g} / \mathrm{mL}$, respectively. Daptomycin exerted the highest antibacterial activity; the number of resistant strains was $0 \%$ (susceptibility: $\leq 1 \mu \mathrm{g} / \mathrm{mL}$ ). Additionally, while not Phase III data, MRSA MICs results (100 isolates derived from blood and 200 isolates derived from skin-related tissues) isolated in Japan showed almost the same $\mathrm{MIC}_{50}$ and $\mathrm{MIC}_{90}$ results as the Phase III study; daptomycin $\mathrm{MIC}_{90}$ was $1 \mu \mathrm{g} / \mathrm{mL}$ (range $0.25-1 \mu \mathrm{g} / \mathrm{mL}$ ), regardless of the isolated parts. Hence, the distribution of daptomycin MIC values among Japanese MRSA was not wide $(0.25-1 \mu \mathrm{g} / \mathrm{mL})$ and it showed good susceptibility against MRSA isolated in Japan.

\section{Efficacy for ABSSSI}

In the Phase III trial, 73 and 74 patients of 110 ABSSSI patients were eligible for efficacy (end-of-cure and test-ofcure) analysis. Among 73 evaluated patients, the clinical efficacy (end-of-cure) of daptomycin was $85.2 \%$ (46 of 54 patients), compared with $84.2 \%$ (16 of 19 patients) for the vancomycin group. In the microbiological efficacy analysis, 24 of 55 patients (43.6\%) who received daptomycin showed efficacy and 9 of 19 patients (47.4\%) who received vancomycin showed efficacy. Additionally, among 74 evaluated patients, the clinical efficacy (test-of-cure) of daptomycin was $81.8 \%$ (45 of 55 patients) compared with $84.2 \%$ (16 of 19 patients) for the vancomycin group. In the microbiological efficacy analysis, 31 of 55 patients (56.4\%) who received daptomycin showed efficacy and 9 of 19 patients $(47.4 \%)$ who received vancomycin showed efficacy. Especially in the patients with MRSA infections from trauma, burn and post-surgical skin and soft tissue infections, daptomycin and vancomycin had 81.6\% (31 of 38 patients) and $84.6 \%$ (11 of 13 patients) clinical 
efficacy, respectively. On the other hand, for the patients with infected ulcers and ulceration, daptomycin and vancomycin showed 100\% (9 of 9 patients) and 80\% (4 of 5 patients) clinical efficacy, respectively.

\section{Efficacy for bacteremia}

Among 11 bacteremia patients, four patients were eligible for efficacy analysis. There were no endocarditis patients. Among four patients, end-of-cure clinical and microbiological success rates were $50 \%$ (2/4 patients) and $100 \%$ (4/4 patients), respectively. Test-of-cure clinical and microbiological success rates were both $50 \%$ (2/4 patients). Compared with another clinical study, while the number of patients was low in the Japanese Phase III trial, daptomycin showed similar efficacy with non-Japanese bacteremia patients infected with MRSA. ${ }^{45}$

Therefore, this Phase III clinical trial demonstrated that daptomycin is as effective as standard therapy for the treatment of ABSSSI, and daptomycin was also effective in the treatment of bacteremia associated with MRSA infections in Japanese as well as non-Japanese patients. ${ }^{45,46}$

\section{Safety and tolerability}

Across the studies, 99 and 22 patients who received daptomycin and vancomycin, respectively, were enrolled in safety analysis. During the Phase III trial, daptomycin was generally well tolerated; 23 of 99 daptomycin-treated patients $(23.2 \%)$ experienced adverse events considered to be related to study treatment; the major adverse events were composed of elevation of aspartate aminotransferase (seven patients, 7.1\%), alanine aminotransferase (seven patients, 7.1\%), alkaline phosphatase (two patients, $2.0 \%$ ), CPK (two patients, 2.0\%), eosinophil (two patients, $2.0 \%$ ) levels, eczema (two patients, 2.0\%), fever (two patients, $2.0 \%$ ), diarrhea (two patients, $2.0 \%$ ), and decreasing platelet level (two patients, 2.0\%). The frequency and distribution of adverse effects were similar in both treatment groups; six of 22 patients $(27.3 \%)$ of comparator agent treated experienced adverse effects. While in the early trials of daptomycin use, skeletal muscle toxicity was observed frequently, as measured by elevations of the CPK muscle isoenzyme, in the Japanese Phase III trial, no patient experienced severe elevation of CPK. Treatment discontinuations due to adverse effects related to treatment drugs occurred for three patients $(3 \%)$ in the daptomycin group and two patients $(9.1 \%)$ in the comparator group. Two patients in each treatment group died during the study; none of the deaths were considered to be treatment-related.

\section{Non-Japanese studies}

As daptomycin has been approved for use in Japan for less than one year, there is limited clinical, in vivo, and in vitro data to support using the drug. However, daptomycin has been investigated in other countries. Safdar et al showed that $\mathrm{AUC}_{0-24} / \mathrm{MIC}$ and maximum concentration/ MIC showed best correlation with antibacterial activity with an in vivo study and daptomycin also had a long lasting postantibiotic effect (PAE) of 5 hours activity against $S$. aureus, including MRSA and VISA, with daptomycin MIC 0.125 to $4 \mu \mathrm{g} / \mathrm{mL}$ using an in vitro pharmacodynamic model simulating human exposure ( 0 to $9 \mathrm{mg} / \mathrm{kg}$ ). Consequently, model fitting resulted in an $r^{2}$ of $>0.80$ for all isolates showing significant bactericidal activity $\left(\mathrm{ED}_{80}\right)$ against these isolates with daptomycin dose ranging between 3 and $7 \mathrm{mg} / \mathrm{kg}$. Additionally, daptomycin has been demonstrated to have a rapid bactericidal effect against organisms with elevated daptomycin MICs, including VISA. ${ }^{48}$

The addition of rifampicin to daptomycin also enhanced bacterial killing in an animal study with MRSA experimental aortic valve endocarditis. ${ }^{49}$ The activity of the combination therapies and dual combination was evaluated in rats with MRSA experimental aortic valve endocarditis. ${ }^{49}$ As a result, the combination of daptomycin and rifampicin produced a lower remaining bacterial vegetation density at the end of therapy than daptomycin alone $\left(2.9 \pm 0.8 \mathrm{vs} 4.6 \pm 1.6 \log _{10}\right.$ cfu/g vegetation; $P=0.006)$. Daptomycin was also evaluated alone and in combination with rifampicin in a guinea pig foreign-body MRSA infection model. ${ }^{50}$ After 4 days of treatment, daptomycin monotherapy eradicated the infection in 4/12 (33\%) animals, with daptomycin and rifampicin resulting in a $67 \%$ cure rate. Likewise, daptomycin sub-MICs combined with gentamicin concentrations lower than the MIC yielded synergy in 34 (68\%) of the $50 \mathrm{~S}$. aureus strains, as well as between daptomycin and various $\beta$-lactams against MRSA. ${ }^{46}$ Animal studies models of $S$. aureus endocarditis vegetations suggested that adding gentamicin to daptomycin dosed at either 6 or $10 \mathrm{mg} / \mathrm{kg} /$ day potentiated bactericidal activity, generally more so than rifampicin. ${ }^{49,51}$ While there are no published randomized clinical trials comparing the combination of daptomycin alone to daptomycin-aminoglycoside in patients with serious MRSA infections, vancomycingentamicin was numerically inferior to daptomycin alone in the treatment of MRSA bacteremia and endocarditis in a randomized trial, although statistical significance was not achieved (14 [31.8] of 44 vs 20 [44.4\%] of 45 patients; $P=0.28) .^{45,52}$ 
Additionally, daptomycin is active in the static and growth phases of bacteria, ${ }^{46,53}$ a property that is highly unusual. While biofilm formation is an important virulence factor that allows bacteria to resist host responses and antibacterial agents, in vitro activities of several antibiotics against two $S$. aureus with pharmacokinetic-pharmacodynamic model, the combination of daptomycin and rifampicin was also bactericidal against biofilm-produced MRSA isolates, achieving the limit of detection at 72 hours.

\section{Patient-focused perspectives such as patient satisfaction and acceptability}

One of the major drawbacks of daptomycin is its lack of effectiveness to treat pulmonary infections since surfactants render it ineffective. Silverman et al found that the interaction of daptomycin with pulmonary surfactant ${ }^{41}$ in an animal pneumonia model exhibited an unusual pattern of activity, although daptomycin has failed to meet statistical noninferiority criteria in a clinical trial for severe community-acquired pneumonia, compared to ceftriaxone. ${ }^{40}$

Hanberger et al evaluated the influence of $\mathrm{Ca}^{2+}$ and albumin on daptomycin with $S$. aureus in an in vitro study. Consequently, although the MICs of daptomycin was not influenced by different $\mathrm{Ca}^{2+}$ concentrations, MICs for $S$. aureus were lowered and PAE were prolonged with increasing concentrations of $\mathrm{Ca}^{2+}$ in the broth. Herein, daptomycin antibacterial activity could be important to ascertain a physiologic free $\mathrm{Ca}^{2+}$ concentration. ${ }^{54}$

Compared with healthy volunteers, clearance in subjects on dialysis is approximately one-third of that in nondialysis subjects $(0.27$ vs $0.81 \mathrm{~L} /$ hour $) .{ }^{55}$ While there is no dose adjustment recommended for patients with renal impairment and creatinine clearance $>30 \mathrm{~mL} /$ minute, in patients with $<30 \mathrm{~mL} / \mathrm{minute}$, the dose recommendation is $4 \mathrm{mg} / \mathrm{kg}$ every 48 hours in patients on hemodialysis or continuous ambulatory peritoneal dialysis, dose administration is recommended postdialysis on dialysis days. ${ }^{56}$ Additionally, in patients with mild-to-moderate hepatic impairment, the pharmacokinetics of daptomycin are not significantly altered and no dosage adjustment is necessary.

Moreover, some studies suggested that treatment with vancomycin was associated with the development of vancomycin heterogeneous resistance and accompanied by daptomycin heteroresistance. ${ }^{36}$ Hence, patients heavily exposed to vancomycin prior to treatment with daptomycin may be at increased risk of a suboptimal response to daptomycin if a nonsusceptible subpopulation emerges.
As the results of early trials of daptomycin revealed, patients should be observed for development of muscle pain or weakness and have weekly CPK levels determined, which may require more frequent monitoring in those with renal insufficiency or who are receiving concomitant statin therapy. However, elevations in CPK are rarely treatmentlimiting. In a prospective trial conducted in foreign country comparing daptomycin with conventional treatment for bacteremia and endocarditis, CPK elevations occurred in $6.7 \%$ of patients in the daptomycin arm compared with $0.9 \%$ in patients in the standard therapy group; ${ }^{45}$ in most patients, CPK levels returned to normal during treatment. Additionally, there have been case reports of rhabdomyolysis during daptomycin use; it has also been observed in patients on concomitant treatment with 3-hydroxy-3methylglutaryl-coenzyme A (HMG-CoA) reductase inhibitors. ${ }^{57}$ Additionally, daptomycin has been shown to interact with thromboplastin reagents and cause a falsely elevated International Normalized Ratio. ${ }^{58}$

Furthermore, several case reports of daptomycin that include eosinophilic pneumonia have been described although the mechanisms was not known. ${ }^{59}$ The pharmacokinetics, safety, and efficacy of daptomycin in children have not been established. Daptomycin is coded category B for pregnancy. Lastly, daptomycin is not compatible with dextrose-containing diluents due to incompatibilities.

\section{Conclusions}

Increasing treatment choices for MRSA infection, vancomycin's shortcomings, and the emergence of new MRSA strains with lower susceptibility against vancomycin are changing perceptions. The Japanese Phase III clinical trial data of daptomycin show that the highest susceptibility compared with other anti-MRSA agents and the efficacy and safety of daptomycin are similar to those of vancomycin for the treatment of ABSSSI and were similar in other clinical studies conducted in foreign countries. ${ }^{46}$ These data suggest that daptomycin has been evaluated with promising results in MRSA bacteremia and ABSSSI.

Daptomycin has a rapid bactericidal activity associated with its unique mechanism of action on the cell membrane. Hence, it might result in more rapid clinical resolution of infections. Additionally, some clinical studies showed that combination therapies were effective for endocarditis and utility in orthopedic infections deserves early investigation, as a combination of cidality and activity against biofilms suggests potential. ${ }^{47-50}$ Furthermore, while daptomycin has favorable pharmacokinetic properties 
allowing once-daily dosing and toxicity is rare with the currently recommended dosing, some in vitro studies suggest daptomycin has the potential for cross-resistance with vancomycin. ${ }^{36,37}$

In summary, daptomycin is the first available agent from a new class of antibiotics, the cyclic lipopeptides. It has antibiotic activity against a wide range of Gram-positive organisms. Especially for MRSA, daptomycin showed higher activity than other anti-MRSA agents. The Japanese Phase III clinical trial demonstrated that daptomycin given at $4 \mathrm{mg} / \mathrm{kg}$ and $6 \mathrm{mg} / \mathrm{kg}$ once per day is a safe and effective standard therapy for the treatment of ABSSSI and bacteremia caused by MRSA, but side effects remain to be evaluated in large-scale trials.

\section{Disclosure}

The authors report no conflicts of interest in this work.

\section{References}

1. Klein E, Smith DL, Laxminarayan R. Hospitalizations and deaths caused by methicillin-resistant Staphylococcus aureus, United States, 1999-2005. Emerg Infect Dis. 2007;13:1840-1846.

2. Japan Nosocomial Infections Surveillance [homepage on the Internet]. Japan Nosocomial Infections Surveillance Report. Available from: http://www.nih-janis.jp/report/open_report/2010/2/1/ken_Open_ Report_201004.pdf. Accessed February 7, 2012. Japanese.

3. National Nosocomial Infections Surveillance. National Nosocomial Infections Surveillance system report, data summary from January 1992 through June 2004. Am Infect Control. 2004;32:470-485.

4. Howden BP, Ward PB, Charles PG, et al. Treatment outcomes for serious infections caused by methicillin-resistant Staphylococcus aureus with reduced vancomycin susceptibility. Clin Infect Dis. 2004;38:521-528

5. Charles PG, Ward PB, Johnson PD, Howden BP, Grayson ML. Clinical features associated with bacteremia due to heterogeneous vancomycin-intermediate Staphylococcus aureus. Clin Infect Dis. 2004;38:448-451.

6. Steinkraus G, White R, Friedrich L. Vancomycin MIC creep in nonvancomycin-intermediate Staphylococcus aureus (VISA), vancomycinsusceptible clinical methicillin-resistant $S$. aureus (MRSA) blood isolates from 2001-2005. J Antimicrob Chemother. 2007;60:788-794.

7. Takesue Y, Nakajima K, Takahashi Y, et al. Clinical characteristics of vancomycin minimum inhibitory concentration of $2 \mu \mathrm{g} / \mathrm{mL}$ methicillinresistant Staphylococcus aureus strains isolated from patients with bacteremia. J Infect Chemother. 2011;17:52-57.

8. Hagihara M, Wiskirchen DE, Kuti JL, Nicolau DP. In vitro pharmacodynamics of vancomycin and cefazolin alone and in combination against methicillin-resistant Staphylococcus aureus. Antimicrob Agents Chemother. 2012;56:202-207.

9. Hatano K, Yokota Y, Hanaki H, Sunakawa K. Combined effect of vancomycin or teicoplanin plus a $\beta$-lactam antibiotic in mouse infection models caused by $\beta$-lactam antibiotic induced vancomycin-resistant MRSA (BIVR). Kansenshogaku Zasshi. 2006;80:243-250.

10. Hanaki H, Yamagushi Y. Epidemiological investigation of " $\beta$-lactam antibiotic induced vancomycin-resistant MRSA (BIVR)". Kansenshogaku Zasshi. 2003;77:499-504.

11. Saito N, Aoki K, Sakurai T, et al. Linezolid treatment for intracranial abscesses caused by methicillin-resistant Staphylococcus aureus - two case reports. Neurol Med Chir. 2010;50:515-517.
12. Endimiani A, Blackford M, Dasenbrook EC, et al. Emergence of linezolid-resistant Staphylococcus aureus after prolonged treatment of cystic fibrosis patients in Cleveland, Ohio. Antimicrob Agents Chemother. 2011;55:1684-1692.

13. Dumitrescu O, Boisset S, Badiou C, et al. Effect of antibiotics on Staphylococcus aureus producing Panton-Valentine leukocidin. Antimicrob Agents Chemother. 2007;5:1515-1519.

14. Dumitrescu O, Badiou C, Bes M, et al. Effect of antibiotics, alone and in combination, on Panton-Valentine leukocidin production by a Staphylococcus aureus reference strain. Clin Microbiol Infect. 2008; $14: 384-388$

15. Smith K, Gemmell CG, Hunter IS. The association between biocide tolerance and the presence or absence of qac genes among hospitalacquired and community-acquired MRSA isolates. J Antimicrob Chemother. 2008;61:78-84.

16. Yabe S, Takano T, Higuchi W, Mimura S, Kurosawa Y, Yamamoto T. Spread of the community-acquired methicillin-resistant Staphylococcus aureus USA300 clone among family members in Japan. $J$ Infect Chemother. 2010;16:372-374.

17. Moran GJ, Krishnadasan A, Gorwitz RJ, et al. Methicillin-resistant $S$. aureus infections among patients in the emergency department. N Engl J Med. 2006;17;355:666-674.

18. Small PM, Chambers HF. Vancomycin for Staphylococcus aureus endocarditis in intravenous drug users. Antimicrob Agents Chemother. 1990;34:1227-1231.

19. Bernareggi A, Borghi A, Borgonovi M, et al. Teicoplanin metabolism in humans. Antimicrob Agents Chemother. 1992;36: $1744-1749$.

20. Hagihara M, Umemura T, Kimura M, Mori T, Hasegawa T, Mikamo H. Exploration of optimal teicoplanin dosage based on pharmacokinetic parameters for the treatment of intensive care unit patients infected with methicillin-resistant Staphylococcus aureus. J Infect Chemother. 2011. [Epub ahead of print.]

21. Kanazawa N, Matsumoto K, Ikawa K, Fukamizu T, Shigemi A, Yaji K. An initial dosing method for teicoplanin based on the area under the serum concentration time curve required for MRSA eradication. J Infect Chemother. 2011;17:297-300.

22. Cavalcanti AB, Goncalves AR, Almeida CS, Bugano DD, Silva E. Teicoplanin versus vancomycin for proven or suspected infection. Cochrane Database Syst Rev. 2010;16:CD007022.

23. Matsumoto K, Takeshita A, Ikawa K, et al. Higher linezolid exposure and higher frequency of thrombocytopenia in patients with renal dysfunction. Int J Antimicrob Agents. 2010;36:179-181.

24. Beibei L, Yun C, Mengli C, Nan B, Xuhong Y, Rui W. Linezolid versus vancomycin for the treatment of gram-positive bacterial infections: meta-analysis of randomised controlled trials. Int J Antimicrob Agents. 2010;35:3-12.

25. Tanigawara Y, Sato R, Morita K, Kaku M, Aikawa N, Shimizu K. Population pharmacokinetics of Arbekacin in patients infected with methicillin-resistant Staphylococcus aureus. Antimicrob Agents Chemother. 2006;50:3754-3762.

26. Oleson FB Jr, Berman CL, Kirkpatrick JB, Regan KS, Lai JJ, Tally FP. Once-daily dosing in dogs optimizes daptomycin safety. Antimicrob Agents Chemother. 2000;44:2948-2953.

27. Silverman JA, Perlmutter NG, Shapiro HM. Correlation of daptomycin bactericidal activity and membrane depolarization in Staphylococcus aureus. Antimicrob Agents Chemother. 2003;47: 2538-2544.

28. Alborn WE Jr, Allen NE, Preston DA. Daptomycin disrupts membrane potential in growing Staphylococcus aureus. Antimicrob Agents Chemother. 1991;35:2282-2287.

29. Hobbs JK, Miller K, O'Neill AJ, Chopra I. Consequences of daptomycin-mediated membrane damage in Staphylococcus aureus. J Antimicrob Chemother. 2008;62:1003-1008.

30. King A, Phillips I. The in vitro activity of daptomycin against 514 Gram-positive aerobic clinical isolates. J Antimicrob Chemother. 2001;48:219-223. 
31. Goldstein EJ, Citron DM, Merriam CV, Warren YA, Tyrrell KL, Fernandez HT. In vitro activities of daptomycin, vancomycin, quinupristin-dalfopristin, linezolid, and five other antimicrobials against 307 gram-positive anaerobic and 31 Corynebacterium clinical isolates. Antimicrob Agents Chemother. 2003;47:337-341.

32. Friedman L, Alder JD, Silverman JA. Genetic changes that correlate with reduced susceptibility to daptomycin in Staphylococcus aureus. Antimicrob Agents Chemother. 2006;50:2137-2145.

33. Julian K, Kosowska-Shick K, Whitener C, et al. Characterization of a daptomycin-nonsusceptible vancomycin-intermediate Staphylococcus aureus strain in a patient with endocarditis. Antimicrob Agents Chemother. 2007;51:3445-3448.

34. Muthaiyan A, Silverman JA, Jayaswal RK, et al. Transcriptional profiling reveals that daptomycin induces the Staphylococcus aureus cell wall stress stimulon and genes responsive to membrane depolarization. Antimicrob Agents Chemother. 2008;52:980-990.

35. Cui L, Tominaga E, Neoh HM, Hiramatsu K. Correlation between reduced daptomycin susceptibility and vancomycin resistance in vancomycin-intermediate Staphylococcus aureus. Antimicrob Agents Chemother. 2006;50:1079-1082.

36. Sakoulas G, Alder J, Thauvin-Eliopoulos C, Moellering RC Jr, Eliopoulos GM. Induction of daptomycin heterogeneous susceptibility in Staphylococcus aureus by exposure to vancomycin. Antimicrob Agents Chemother. 2006;50:1581-1585.

37. Patel JB, Jevitt LA, Hageman J, McDonald LC, Tenover FC. An association between reduced susceptibility to daptomycin and reduced susceptibility to vancomycin in Staphylococcus aureus. Clin Infect Dis. 2006;42:1652-1653.

38. Eisenstein BI. Lipopeptides, focusing on daptomycin, for the treatment of Gram-positive infections. Expert Opin Investig Drugs. 2004;13:1159-1169.

39. Oleson FB, Berman CL, Li AP. An evaluation of the P450 inhibition and induction potential of daptomycin in primary human hepatocytes. Chem Biol Interact. 2004;150:137-147.

40. Pertel PE, Bernardo P, Fogarty C, et al. Effects of prior effective therapy on the efficacy of daptomycin and ceftriaxone for the treatment of community-acquired pneumonia. Clin Infect Dis. 2008;46:1142-1151.

41. Silverman JA, Mortin LI, Vanpraagh AD, Li T, Alder J. Inhibition of daptomycin by pulmonary surfactant: in vitro modeling and clinical impact. J Infect Dis. 2005;191:2149-2152.

42. Cubicin ${ }^{\circledR}$ Summary of Product Characteristics. Available from: http:// www.mrsa-cubicin.jp/secure/practitioner_download/index.html. Accessed February 7, 2012. Japanese.

43. Aikawa N, Kusachi S, Mikamo H, Takesue Y, Watanabe S, Toshinari T. Efficacy of daptomycin in Japanese patients with MRSA infection in relation to the susceptibility of baseline isolates in a randomized study. Poster A2-020. Chicago, IL: 51st Interscience Conference on Antimicrobial Agents and Chemotherapy. American Society for Microbiology; 2011.

44. Clinical and Laboratory Standards Institute. Methods for dilution antimicrobial susceptibility tests for bacteria that grow aerobically. In: Approved Standards - Eighth Edition. CLSI document M07-A8. Wayne, PA: Clinical and Laboratory Standards Institute; 2009.

45. Fowler VG Jr, Boucher HW, Corey GR, et al; S. aureus Endocarditis and Bactevenia Study Group. Daptomycin versus standard therapy for bacteremia and endocarditis caused by Staphylococcus aureus. N Engl J Med. 2006;355:653-665.

Therapeutics and Clinical Risk Management

\section{Publish your work in this journal}

Therapeutics and Clinical Risk Management is an international, peerreviewed journal of clinical therapeutics and risk management, focusing on concise rapid reporting of clinical studies in all therapeutic areas, outcomes, safety, and programs for the effective, safe, and sustained use of medicines. This journal is indexed on PubMed Central, CAS,
46. Rand KH, Houck HJ. Synergy of daptomycin with oxacillin and other beta-lactams against methicillin-resistant Staphylococcus aureus. Antimicrob Agents Chemother. 2004;48:2871-2875.

47. Safdar N, Andes D, Craig WA. In vivo pharmacodynamic activity of daptomycin. Antimicrob Agents Chemother. 2004;48:63-68.

48. Cha R, Grucz RG Jr, Rybak MJ. Daptomycin dose-effect relationship against resistant gram-positive organisms. Antimicrob Agents Chemother. 2003;47:1598-1603.

49. Sakoulas G, Eliopoulos GM, Alder J, et al. Efficacy of daptomycin in experimental endocarditis due to methicillin-resistant Staphylococcus aureus. Antimicrob Agents Chemother. 2003;47:1714-1718.

50. John AK, Baldoni D, Haschke M, et al. Efficacy of daptomycin in implant-associated infection due to methicillin-resistant Staphylococcus aureus: importance of combination with rifampin. Antimicrob Agents Chemother. 2009;53:2719-2724.

51. Tsuji BT, Rybak MJ. Short-course gentamicin in combination with daptomycin or vancomycin against Staphylococcus aureus in an in vitro pharmacodynamic model with simulated endocardial vegetations. Antimicrob Agents Chemother. 2005;49:2735-2745.

52. Rehm SJ, Boucher H, Levine D, et al. Daptomycin versus vancomycin plus gentamicin for treatment of bacteraemia and endocarditis due to Staphylococcus aureus: subset analysis of patients infected with methicillin-resistant isolates. J Antimicrob Chemother. 2008;62:1413-1421.

53. LaPlante KL, Rybak MJ. Impact of high-inoculum Staphylococcus aureus on the activities of nafcillin, vancomycin, linezolid, and daptomycin, alone and in combination with gentamicin, in an in vitro pharmacodynamic model. Antimicrob Agents Chemother. 2004;48:4665-4672.

54. Hanberger H, Nilsson LE, Maller R, Isaksson B. Pharmacodynamics of daptomycin and vancomycin on Enterococcus faecalis and Staphylococcus aureus demonstrated by studies of initial killing and postantibiotic effect and influence of $\mathrm{Ca} 2+$ and albumin on these drugs. Antimicrob Agents Chemother. 1991;35:1710-1716.

55. Dvorchik B, Arbeit RD, Chung J, Liu S, Knebel W, Kastrissios H. Population pharmacokinetics of daptomycin. Antimicrob Agents Chemother. 2004;48:2799-2807.

56. Cubist Pharmaceuticals Inc. Daptomycin: full prescribing information. Available from: http://www.cubicin.com/pdf/PrescribingInformation. pdf. Accessed February 10, 2012.

57. Odero RO, Cleveland KO, Gelfand MS. Rhabdomyolysis and acute renal failure associated with the co-administration of daptomycin and an HMG-CoA reductase inhibitor. J Antimicrob Chemother. 2009;63:1299-1300.

58. Webster PS, Oleson FB Jr, Paterson DL, et al. Interaction of daptomycin with two recombinant thromboplastin reagents leads to falsely prolonged patient prothrombin time/International Normalized Ratio results. Blood Coagul Fibrinolysis. 2008;19:32-38.

59. Falagas ME, Rafailidis PI, Kasiakou SK, et al. Effectiveness and nephrotoxicity of colistin monotherapy vs colistin-meropenem combination therapy for multidrug-resistant Gram-negative bacterial infections. Clin Microbiol Infect. 2006;12:1227-1230.

\section{Dovepress}

EMBase, Scopus and the Elsevier Bibliographic databases. The manuscript management system is completely online and includes a very quick and fair peer-review system, which is all easy to use. Visit $\mathrm{http}: / / \mathrm{www}$. dovepress.com/testimonials.php to read real quotes from published authors. 